Edith Cowan University

Research Online

ECU Publications 2013

$1-1-2013$

\title{
Psychological resilience of Western Australian police officers: relationship between resilience, coping style, psychological functioning and demographics
}

\author{
Gemma M. Balmer \\ Edith Cowan University \\ Julie Ann Pooley \\ Edith Cowan University \\ Lynne Cohen \\ Edith Cowan University
}

Follow this and additional works at: https://ro.ecu.edu.au/ecuworks2013

Part of the Psychiatry and Psychology Commons

10.1080/15614263.2013.845938

Balmer, G. M., Pooley, J. , \& Cohen, L. (2013). Psychological resilience of Western Australian police officers:

relationship between resilience, coping style, psychological functioning and demographics. Police Practice and Research: An International Journal, 15(4), 270-282.

This is an Accepted Manuscript of an article published by Taylor \& Francis in Police Practice and Research, on 10 Oct 2013, available online: here.

This Journal Article is posted at Research Online.

https://ro.ecu.edu.au/ecuworks2013/777 
PSYCHOLOGICAL RESILIENCE OF WESTERN AUSTRALIAN POLICE OFFICERS:

RELATIONSHIP BETWEEN RESILIENCE, COPING STYLE, PSYCHOLOGICAL

FUNCTIONING AND DEMOGRAPHICS.

\author{
Gemma M. Balmer \\ Julie Ann Pooley * \\ Lynne Cohen \\ Edith Cowan University
}

\begin{abstract}
This study examines the relationships between resilience, coping style, psychological functioning and the demographic variables of gender, age, rank and length of service in a sample of 285 Western Australian Police officers. Regression analysis indicated that resilience was predicted by greater use of rational coping and less use of emotional coping, but not psychological functioning. Increased age, rank and length of service were all correlated with significantly lower resilience scores. Significant differences in coping styles were found for all demographic variables. Implications of these findings and recommendations for future research are discussed.
\end{abstract}

Keywords: psychological resilience, police, coping, psychological functioning 
* Correspondence should be forwarded to Julie Ann Pooley:

Julie Ann Pooley PhD

Edith Cowan University

School of Psychology and Social Science

270 Joondalup Dve, Joondalup.

Perth 6027 WA, AUSTRALIA

Email: j.pooley@ecu.edu.au Ph: 61863045591

Author Biography

Gemma Balmer has completed this study as partial fullfillment of Master of Clinical Psychology at Edith Cowan University and currently works as a counsellor in a nongovernment agency providing Employee Assistance Program services.

Associate Professor Julie Ann Pooley is Associate Dean Teaching and Learning and leads the Lifespan Resilience Research Group located in Edith Cowan University’s School of Psychology and Social Science., Faculty Computing Health and Science. Her research interest's areas centre on the development of the resilience concept, its measurement and posttraumatic growth.

Professor Lynne Cohen is Executive Dean, Faculty of Education and Arts and Pro-Vice Chancellor at Edith Cowan University. She is a community psychologist and has taught undergraduate and postgraduate programs in psychology. She is responsible for establishing the Lifespan Resilience Research Centre where she leads a team of researchers at ECU to develop innovative, evidence based practices that enhance mental health and build resilience across the human lifespan. 


\section{Acknowledgements}

The authors would like to acknowledge the support of the Western Australian Police and the police officers who participated in the research.

\section{INTRODUCTION}

Policing is recognised as one of the most stressful and high risk occupations in Australia (Mayhew, 2001). In the line of duty, police officers are repeatedly exposed to operational stressors in the form of unpredictable and potentially dangerous or traumatic situations (Hickman, Fricas, Strom, \& Pope, 2011). Organisational characteristics of law enforcement agencies including shift work, lack of resources, staff shortages, lack of managerial support and bureaucratic procedures are also recognised as sources of stress for police officers (Martinussen, Richardsen, \& Burke, 2007). Police research has comprehensively established that if not managed effectively, stress has a significant negative impact on the physical health and psychological functioning of police officers (LeBlanc, Regehr, Jelley, \& Barath, 2008). Whilst Police officers are considered at greater risk for developing physical health problems including cardiovascular disease and high blood pressure (Franke, Ramey, \& Shelley, 2002), post-traumatic stress disorder (PTSD), depression and insomnia (LeBlanc et al., 2008) and increased consumption of alcohol, tobacco and illicit substances (Kohan \& O’Connor, 2002) limited research has attempted to determine why the majority of officers who witness traumatic or life-threatening events do not experience significant impairment or develop psychopathology such as PTSD, (Bonanno, 2004).

Following a move away from problem-orientated research to a strengths-based line of enquiry, attention has been directed toward the concept of psychological resilience in an attempt to explain why many individuals are able to endure traumatic, life-threatening and 
stressful events without experiencing psychological impairment or significant disruption to normal functioning (Richardson, 2002).

\section{LITERATURE REVIEW}

Fundamentally, psychological resilience is defined as the ability to successfully cope and adapt when faced with adversity or stressful life events (Pooley \& Cohen, 2010). Thus, in the context of this study, police officers who have been exposed to stressful or traumatic situations and have not experienced significant impairment or developed psychological disorders such as depression or PTSD can be defined as being resilient.

Resilience is a complex, multidimensional construct in which there is no single specific characteristic or trait that makes an individual resilient, rather there are multiple pathways to resilience which are different for every person and vary depending on their circumstance (Newman, 2005). Resilient people share a number of defining attributes (protective factors), and the presence or absence of these attributes account for the variation in individuals’ resilience responses to stressful life events (Diehl \& Hay, 2010). The defining attributes of resilience, can be classified into dispositional attributes, family support and cohesion and external support systems (Frigborg, Hjemdal, Rosenvinge, \& Martinussen, 2003). Resilient people are characterised as having favourable dispositional attributes including high self-esteem and self-efficacy, positive self-image, internal locus of control, optimism for the future, sense of control and competence in their life, and use of active coping strategies (Burke, Shakespeare-Finch, Paton, \& Ryan, 2006; Diehl \& Hay, 2010). In relation to support systems, resilient individuals have a history of family stability and cohesiveness, and they engage in and reciprocate social support with others (EarvolinoRamirez, 2007).

The protective factors described above have been consistently reported in literature investigating resilience reactions of the general population however, police populations are 
markedly different from the general population, as police officers experience repeated exposure to stressful and potentially traumatic events while on duty (Anderson, Litzenberger, \& Plecas, 2002). Thus, raising the question as to whether resilience is different for police officers.

\section{Coping Styles}

Coping strategies have been identified as a contributing factor in the development and maintenance of resilience during periods of stress or trauma (Hoge, Austin, \& Pollack, 2007). Coping is defined as an individual's cognitive and behavioural actions to manage internal or external stressors or problems which exceed their personal resources. Folkman and Lazarus (1980) classified coping strategies as either problem-focused or emotion-focused coping. Problem-focused coping defines efforts to modify or eliminate stressors through direct action; whereas emotion-focused coping strategies attempt to alleviate the emotional reactions to stressors through cognitive efforts to change the meaning of the situation (Matud, 2004). As an alternative to problem-focused and emotion-focused coping styles, coping behaviour has also been described as being approach-based and avoidance-based (Ben-Zur, 2009).

It is generally accepted that most approach-based coping strategies correspond with problem-focused coping (adaptive and associated with positive outcomes) and avoidancebased coping equates with emotion-focused coping (maladaptive and associated with negative outcomes ) (Beasley, Thompson, \& Davidson, 2003; Pooley, Cohen, O’Connor \& Taylor, 2012). Research investigating the coping behaviour of police officers generally found that officers proactively utilise problem-focused coping strategies to resolve occupational stress but do not effectively use emotion-focused coping strategies to deal with their emotional reactions to stress (Alexander \& Walker, 1994; Biggam, Powerr, \& MacDonald, 1997a).

\section{Impact of Coping Styles on Resilience}


As an individual's ability to moderate the effects of stressful and traumatic events is influenced by the coping style they employ (Ben-Zur, 2009), it is hypothesised that coping style also has an impact on resilience level. While the effect of coping styles on physical and psychosocial functioning has received significant attention in the literature, few studies have investigated the impact of coping styles on resilience, with an apparent lack of research on police populations.

One of the few studies directly investigating the relationship between coping styles and resilience of police officers was conducted by Pole, Kulkarni, Bernstein and Kaufmann (2006), who examined resilience in a sample of U.S. retired police officers. Resilience was found to be significantly correlated with less reliance on distancing and escape-avoidance coping strategies during times of stress or adversity. These maladaptive coping strategies were hypothesised to reduce resilience by contributing to poorer mental health as officers avoided sharing their experiences with others, particularly family and friends, leading to increased social isolation and maintenance of stress symptoms (Pole et al., 2006).

\section{Impact of Demographics on Resilience and Coping Style}

Consistent with coping behaviour, demographic variables including age, gender, marital status, education level and length of employment have been found to influence an individual's level of resilience. However, research investigating the relationship between demographics variables and resilience of police officers has been limited. The findings from the small number of available studies have provided mixed results and highlight the need for further detailed investigation.

Prati and Pietrantoni (2010) investigated the effect of risk and protective factors on the resilience of a sample of Italian police officers. The study failed to find a significant relationship between gender and resilience; but found that length of service predicted resilience with longer serving officers experiencing the lowest levels of resilience. The effect 
of length of service on resilience was hypothesised to be the result of officer's cumulative exposure to stress and trauma during their career, which negatively impacts their coping abilities and reduces resilience (Prati and Pietrantoni, 2010).

Determining how demographic and occupational characteristics influence coping behaviour would provide a better understanding of which police officers deal most effectively with stress and which officers experience distress due to their reliance on ineffective or maladaptive coping strategies. While this avenue of research would appear to provide valuable information for law enforcement agencies, there has been a reluctance to investigate the impact of demographic and occupational variables on coping behaviour of police officers (Burke \& Mikkelsen, 2005).

An isolated study has attempted to determine whether the demographic and occupational variables of age, gender, marital status, rank and length of service produce differences in the coping behaviour of police officers (Biggam et al., 1997a). With regard to age, the only statistically significant result was that the youngest officers used the emotionfocused coping strategy of seeking social support more than any other age group. This finding corresponds with the expected social behaviour of young people, who typically rely on peers for emotional support to deal with stress. For rank, sergeants were used problemfocused coping strategies significantly more than either constables or higher ranking officers. There was no correlation between coping style and marital status or length of service (Biggam et al., 1997a).

With regard to gender, results pertaining to the impact of gender on coping style have been inconsistent. Biggam et al (1997a) and Alexander and Walker (1994) reported that female Scottish police officers use emotion-focused coping strategy of seeking social support significantly more than male officers. Alexander and Walker (1994) considered the limited use of social support by male officers to reflect the work environment and culture of the 
Scottish police, which promotes problem-focused coping rather than coping through emotional expression. Haarr and Morash (1999) reported no significant differences in the coping behaviour of male and female officers and argued that police culture and training, rather than gender, has the greatest influence on the coping styles employed by officers to manage organisational stressors. These inconsistent results support the need for further investigation to improve the understanding of how demographic and occupational variables influence the coping behaviour of police officers.

Therefore, the purpose of this research is to examine the relationships between resilience, coping styles, self-reported psychological functioning and demographic variables using the following research questions:

1. Does coping style or self-reported psychological functioning predict police officer's level of resilience?

2. Does gender, length of service, rank or age impact police officer's level of resilience?

3. Do the demographic variables of gender, length of service, rank and age influence the coping styles used by police officers?

\section{METHOD}

\section{Participants}

A sample of 293 participants returned completed questionnaires, providing a response rate of $29.3 \%$. Useable data for analysis was collected from 285 participants; 230 males (80.7\%) and 55 females (19.3\%); 8 participants were excluded due to missing data. These are presented in Table 1 below.

[Table 1 here]

\section{Materials}

The questionnaire package contained a cover letter self-addressed return envelope and the four questionnaires as follows: 


\section{Resilience Scale for Adults (RSA)}

The RSA was developed by Friborg et al. (2003), for measuring resilience in adults. The RSA is a self-report 33 itemscale measured on a 5-point positive and neagative semantic differential attribute.. Scores on the RSA range from 33 to 231, with higher scores indicating higher level of resilience. The RSA consists of 6 factors: positive perception of self, planned future, social competence, structured style, family cohesion and social resources. Assessments of the RSA has shown it to have high internal consistency, ranging from 0.67 to 0.90, and satisfactory test-retest reliability ranging from 0.69 to $0.86(\mathrm{p}<0.01)$ (Friborg, Barlaug, Martinussen, Rosenvinge, \& Hjemdal, 2005).

\section{General Health Questionnaire (GHQ-12)}

The GHQ-12 is a brief, 12-item screening instrument for detecting minor psychiatric illness or psychological strain (Goldberg \& Williams, 1988). Participant's responses are recorded using a 4-point Likert scale (scored 0-3), evaluating their psychological wellbeing over the previous few weeks. Scores range from 0-36, with higher scores indicating poorer mental health status and greater distress. The GHQ-12 has a reported Cronbach's alpha coefficient of 0.91 (Kalliath, O’Driscoll \& Brough, 2004)).

\section{Coping Styles Questionnaire (CSQ)}

The 60-item CSQ was constructed to measure coping strategies using a 4-point Likert scale format in which responses are rated as always, often, sometimes, or never The CSQ consists of four scales to measure the four primary coping styles of rational, emotional, avoidance and detached. Test-retest reliability is reported to be $(.74-.85)$ and internal consistency was acceptable (coefficient alphas ranged from .66 - .81) (Roger, Jarvis \& Najarian, 1993).

\section{Procedure}


The participant population was collated from the Western Australian (WA) Police employee database by the WA Police Academic Research Administration Unit. All questionnaire packages were compiled and posted to participants. Participation in the study was voluntary and completed questionnaires were posted back to the researcher.

\section{RESULTS}

The results will be presented in relation to each research question. Prior to analysis, data was examined for outliers and violations of the assumptions. All parametric test assumptions were satisfied.

In order to address the first research question, multiple regression analysis was performed to determine the contribution of rational, detached, emotional and avoidance coping, and psychological functioning on police officers' level of resilience. As shown in Table 2, resilience was significantly predicted by rational and emotional coping styles, but not detached or avoidance coping or psychological functioning. The results indicate that $44.2 \%$ of the variance is explained by rational and emotional coping $\left(\mathrm{R}^{2}=.442, \mathrm{~F}(5,284)=\right.$ 44.23, $\mathrm{p}=.000)$

[Table 2 here]

To address the second research question an independent samples t-test $(\alpha=.05)$ was used to determine whether gender had an impact on officers' level of resilience. Results were not significant, with male officers $(M=130.80, S D=15.25)$ and female officers $(M=$ 132.35, $\mathrm{SD}=16.12)$ reporting similar resilience scores.

To determine whether demographic variables of length of service, rank and age had an impact on level of resilience, a series of one-way analysis of variance (ANOVA, $\alpha=.05$ ) and Tukey HSD post hoc comparisons were conducted. Test assumptions of normality and homogeneity of variance were satisfied. These revealed that a significant effect of years of service for resilience, $F(2,282)=6.42, p=.002$. Post hoc comparisons indicated that officers 
with 1-10 years of service had significantly higher resilience scores than officers with 11-20 years of service or 21 years or more service (see Table 3).

For rank a significant result was obtained, $F(2,281)=5.83, p=.001$. Post hoc comparisons indicated that Constables were significantly more resilient than Senior Constables and Sergeants (see Table 3).

With regard to age, the ANOVA revealed a significant result, $F(2,281)=6.42, p=$ .002. The youngest group of officers aged 18-35 years were significantly more resilient than officers aged 36-45 years and officers aged 46 years and above (see Table 3).

[Table 3 here]

To address the final research question independent samples t-tests $(\alpha=.05)$ and one-way ANOVA $(\alpha=.05)$ with Tukey HSD post hoc comparisons were conducted.

A series of independent samples t-tests $(\alpha=.05)$ were conducted to determine whether gender had an impact on officers coping style. The hypothesis was supported for emotional coping and detached coping (see Table 4).

[Table 4 here]

For Length of service the ANOVA results were statistically significant for all four coping styles (see Table 5).

[Table 5 here]

In regard to Rank (see Table 6) ANOVA results were statistically significant for all four coping styles.

For rational coping, the main post hoc comparisons revealed that Senior Constables relied on rational coping significantly less than Constables and Higher Ranked officers.

For detached coping, the post hoc comparisons revealed that Senior Constables relied on detached coping significantly less than Constables. 
For emotional coping, the post hoc comparisons revealed that Senior Constables used emotional coping significantly more than Constables or Sergeants.

Finally for avoidance coping, the post hoc comparisons revealed that Constables used emotional coping significantly less than Senior Constables or Sergeants.

[Table 6 here]

In regard to age (see Table 7), rational coping was significant, with post hoc comparisons revealing that the officers aged 18-35 years used rational coping significantly more than older officers aged 36-45 years. Emotional coping and avoidance coping were both significant. Post hoc comparisons indicated that officers aged 46 years or older scores significantly higher for emotional and detached coping than officers aged 18-35 years.

[Table 7 here]

\section{DISCUSSION}

Therefore resilience was found to be predicted by greater use of rational coping and less use of emotional coping, but not by psychological functioning. The positive relationship between resilience and rational coping is consistent with the perception that approach-based coping strategies are effective and promote resilience (Herrman et al., 2011). The negative effect of emotional coping on resilience is consistent with Pole et al. (2006), who found that avoidance-based coping strategies negatively impacted on the resilience of a sample of U.S. police officers and suggest that emotion-focused coping is maladaptive and undermines resilience (LeBlanc et al., 2008).

The efficacy to which officers use approach and avoidance-based coping strategies may explain the effects of rational and emotional coping on resilience. Officers reported a preference for approach-based coping strategies, but do not utilise avoidance-based coping effectively to manage their emotional reactions to stress or trauma (Alexander \& Walker, 1994; Biggam et al., 1997a). Police culture and training may reinforce the use of approach- 
based coping at the expense of emotional coping, which denies officers the skills to effectively manage their emotional reactions to the stressors of police work (Evans et al., 1993). Successful regulation of emotional responses to stress are essential for maintaining healthy psychological functioning and resilience (LaRocco, House, \& French, 1980). These findings suggest that to enhance police resilience, it is necessary to improve officers' use of effective emotional coping strategies and promote change within police culture to support the effective expression of emotions.

Psychological functioning (GHQ) did not predict resilience, which is inconsistent with the definition of resilience as a process As there was no significant relationship between resilience and psychological functioning resulting there may be the need for the use of a more detailed psychometric tool to assesspsychological functioning and physical health of police officers. Officers may have also underreported their symptoms in an attempt to maintain an image of healthy physical and psychological functioning.

The lack of gender differences in resilience scores was consistent with the previous findings of resilience in a sample of Italian police officers (Prati \& Pietrantoni, 2010) and the. the influences of police culture and training on officers' perception of stress and coping responses have been proposed to countermand the inherent influences of gender on resilience (Haarr and Morash, 1999). Further research using the RSA is recommended to extend our understanding of gender and resilience of police officers.

Resilience was found to be negatively affected by increased rank, age and length of service and the decline of resilience with increased length of service is consistent with the findings of Prati and Pietrantoni (2010). The decline in officers' resilience with increased length of service has been proposed to result from a cumulative exposure to stressful and

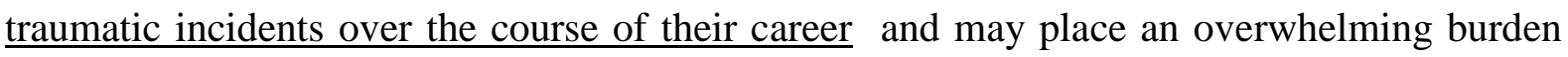
on an officer's coping skills, leading to distress which negatively affects their resilience(Prati 
\& Pietrantoni, 2010). The significant decline in officers' resilience with age was not consistent with previous research findings (Gooding, Hurst, Johnson, \& Tarrier, 2011). The current finding is also not consistent with the definition of resilience. The decline in resilience of older officers suggests that these officers are exposed to additional risk factors unique to policing that undermine their resilience. In particular the decline in resilience with higher ranked officers could be explained by increased organisational stressors stemming from the responsibilities and demands associated with senior rank. Research has previously found that higher ranked officers experience significantly more organisational stressors than lower ranked officers, and that these stressors are associated with poorer physical and psychological health outcomes for senior officers (Biggam, Power, MacDonald, Carcary, \& Moodie, 1997b). This finding suggests that organisational stressors contribute to lower resilience of senior ranked officers by undermining their coping ability and negatively affecting their physical health and psychological functioning.

Resilience of senior ranked officers may also be undermined by a lack of suitable social support networks within the workplace. Effective and meaningful social support systems have been found to moderate the negative effects of occupational stressors by enhancing coping and protecting against physical and psychological harm (Patterson, 2003). Therefore, a lack of access to suitable social support networks by higher ranked officers (see Lord, 1996) may explain the negative relationship between rank and resilience found in the present study. To determine the accuracy of this conclusion, investigation of the differences in access to social support networks across ranks and the effect of organisational stressors and social support on resilience of officers is recommended.

With regard to the third research question, all demographic variables were found to influence the coping styles used by police officers. For gender, both male and female officers reported an overall preference for rational coping and this is consistent with the opinion that 
police culture and training has greater influence than gender on coping behaviour, which reinforces the use of problem-focused and direct-action coping strategies (Haarr and Morash, 1999). However, significant gender differences were found when emotional, detached and avoidance coping styles were considered. Female police officers used emotional coping strategies significantly more than male officers, while male officers relied on detached coping significantly more than female officers. These results are consistent with previous research and suggest that even with police training and culture, gender still has an influence on the coping strategies used by officers (Alexander \& Walker, 1994; Biggam et al., 1997a).

Previously, emotion-based coping strategies have been considered to be maladaptive (Matud 2004, 1995), however, the findings of significantly greater use of emotional coping strategies by female officers and a lack of gender differences in the resilience of officers indicates that emotion-based coping strategies are not necessarily detrimental to officers' wellbeing and resilience. Rather, these results suggest that the use of particular emotionbased coping strategies can be beneficial and enable officers to effectively manage the stressors and organisational demands of policing.

With regard to the demographic variables of length of service, rank and age, the results for all coping styles were consistent across all three demographic variables. In the case of rational and detached coping, a significant decline in the use of these coping styles was found for officers in the middle categories for each demographic variable. The lack of differentiation in all coping styles across demographic variables suggests that other variables are responsible for the patterns of coping behaviour found in this study. Coping behaviour has previously been considered to be influenced by factors including prior learning, life experiences, personality type, self-esteem and perceived level of stress (Diehl \& Hay, 2010). Further investigation of these variables is recommended to determine how the coping styles of police officers change across the course of their career. 
For emotional and avoidance coping, the use of these coping styles were found to increase with longer employment, older age and higher rank. Given that longer serving officers are generally older and hold a rank above Senior Constable, the greater use of emotional and avoidance coping across these demographics is likely the result of factors relating to their longer service history (Lord, 1996). Increased reliance on emotional and avoidance coping strategies by older, longer serving and higher ranked officers may reflect officers' attempts to control their emotional responses to their long history of exposure to stress and trauma (Evans \& Coman, 1993). Thus, if officers' experiences of stress and trauma have a cumulative effect over time, it would be expected that these officers would rely significantly more on emotional or avoidance coping strategies in an attempt to regulate their emotional responses to stressors.

Alternatively, the reliance on emotional and avoidance coping styles by older, longer serving officers may be the consequence of cohort and recruit training differences. Changes in training procedures over time may result in officers developing and using different coping behaviours to manage the effects of stress and trauma, depending when they were recruited and the training they received. Longitudinal investigation of the coping strategies used by police officers across their career would confirm whether the differences in coping found in the present study was the result of cohort differences or if coping changes in response to time or career-related factors.

The current study has a number of limitations. This study did not consider the impact of work roles or work divisions on officer's resilience and coping behaviour. It is possible that work roles influence the type and frequency of exposure to traumatic incidents and stressors, which would have an effect on coping behaviour and resilience. Further research is recommended to ascertain the impact of work role on the development and maintenance of resilience in police populations. Second, the organisational and operational differences across 
law enforcement agencies raise caution in generalizing the research outcomes to allpolice populations.

\section{Conclusion}

In conclusion, this study examined the relationship between resilience, coping styles, self-reported psychological functioning and demographic variables in a sample of Australian police officers. The beneficial outcomes of resilience include the maintenance of healthy physical and psychological functioning, effective coping behaviour and reduction in stress (Newman, 2005). It is expected that further resilience research as recommended from the findings of the current study will improve the understanding of the factors which promote resilience in other police populations. However this research offers to inform law enforcement agencies of the processes and actions that encourage the development of resilience in their officers.

\section{REFERENCES}

Alexander, D. A., \& Walker, L. G. (1994). A study of methods used by Scottish police officers to cope with work-induced stress. Stress Medicine, 10, 131-138.

Anderson, G. S., Litzenberger, R., \& Plecas, D. (2002). Physical evidence of police officer stress. Policing: An International Journal of Police Strategies and Management, 25(2), 399-420.

Beasley, M., Thompson, T., \& Davidson, J. (2003). Resilience in response to life stress: the effects of coping style and cognitive hardiness. Personality and Individual Differences, 34, 77-95.

Ben-Zur, H. (2009). Coping styles and affect. International Journal of Stress Management, 16(2), 87-101. 
Biggam, F. H., Power, K. G., \& MacDonald, R. R. (1997a). Coping with the occupational stressors of police work: A study of Scottish officers. Stress Medicine, 13, 109-115.

Biggam, F. H., Power, K. G., MacDonald, R. R., Carcary, W. B. \& Moodie, E. (1997b). Selfperceived occupational stress and distress in a Scottish police force. Work and Stress, 11(2), 118-133.

Bonanno, G. A., Galea, S., Bucciarelli, A., \& Vlahov, D. (2007). What predicts psychological resilience after disaster? The role of demographics, resources, and life stress. Journal of Consulting and Clinical Psychology, 75(5), 671-682.

Burke, R. J., \& Mikkelsen, A. (2005). Gender differences in policing: Signs of progress? Employee Relations, 27(4), 425-436.

Burke, R. J., Shakespeare-Finch, J., Paton, D., \& Ryan, M. (2006). Characterizing the resilient officer: Individual attributes at point of entry to policing. Traumatology, 12(3), 178-188.

Diehl, M., \& Hay, E. L. (2010). Risk and resilience factors in coping with daily stress in adulthood: The role of age, self-concept incoherence, and personal control. Developmental Psychology,46(5), 1132-1146.

Earvolino-Ramirez, M. (2007). Resilience: A concept analysis. Nursing Forum, 42(2), 7382.

Evans, B. J., Coman, G. J., Stanley, R. O., \& Burrows, G. D. (1993). Police officers’ coping strategies: An Australian police survey. Stress Medicine, 9, 237-246.

Folkman, S., \& Lazarus, R. S. (1980). An analysis of coping in a middle-aged community sample. Journal of Health and Social Behavior, 21, 219-239.

Franke, W. D., Ramey, S. L., \& Shelley, M. C. (2002). Relationship between cardiovascular disease morbidity, risk factors, and stress in a law enforcement cohort. Journal of Occupational and Environmental Medicine, 44, 1182-1189. 
Friborg, O., Hjemdal, O., Rosenvinge, J. H., \& Martinussen, M. (2003). A new rating scale for adult resilience: What are the central protective resources behind healthy adjustment? International Journal of Methods in Psychiatric Research, 12(2), 65-76.

Friborg, O., Barlaug, D., Martinussen, M., Rosenvinge, J. H. \& Hjemdal, O. (2005). Resilience in relation to personality and intelligence. International Journal of Methods in Psychiatric Research, 14(1), 29-42.

Goldberg, D., \& Williams, P. (1988). A user's guide to the General Health Questionnaire. Windsor, UK: NFER Publishing.

Gooding, P. A., Hurst, A., Johnson, J., \& Tarrier, N. (2012). Psychological resilience in young and older adults. International Journal of Geriatric Psychiatry, 27, 262-270.

Haarr, R. N., \& Morash, M. (1999). Gender, race, and strategies of coping with occupational stress in policing. Justice Quarterly, 16(2), 303-335.

Herrman, H., Stewart, D. E., Diaz-Granados, N., Berger, E. L., Jackson, B., \& Yuen, T. (2011). What is resilience? Canadian Journal of Psychiatry, 56(5), 258-265.

Hickman, M. J., Fricas, J., Strom, K. J., \& Pope, M. W. (2011). Mapping police stress. Police Quarterly, 14, 227-250.

Hoge, E. A., Austin, E. D., \& Pollack, M. H. (2007). Resilience: Research evidence and conceptual considerations for posttraumatic stress disorder. Depression and Anxiety, 24, 139-152.

Kalliath, T. J., O’Driscoll, M. P., \& Brough, P. (2004). A confirmatory factor analysis of the General Health Questionnaire-12. Stress and Health, 20, 11-20.

Kohan, A., \& O’Connor, B. P. (2002). Police officer job satisfaction in relation to mood, well-being and alcohol consumption. The Journal of Psychology, 136(3), 307-318. 
LaRocco, J. M., House, J. S., \& French, J. R. P., Jr. (1980). Social support, occupational stress, and health. Journal of Health and Social Behavior, 21, 202- 218.

LeBlanc, V. R., Regehr, C., Jelley, R. B., \& Barath, I. (2008). The relationship between coping styles, performance, and responses to stressful scenarios in police recruits. International Journal of Stress Management, 15(1), 76-93.

Lord, V. B. (1996). An impact of community policing: Reported stressors, social support, and strain among police officers in a changing police department. Journal of Criminal Justice, 24(6), 503-522.

Martinussen, M., Richardsen, A. M., \& Burke, R. J. (2007). Job demands, job resources, and burnout among police officers. Journal of Criminal Justice, 35, 239-249.

Matud, M. P. (2004). Gender differences in stress and coping styles. Personality and Individual Differences, 37, 1401-1415.

Mayhew, C. (2001). Occupational health and safety risks faced by police officers. Trends and Issues in Crime and Criminal Justice, 196, 1-6.

Montgomery, S. B., Wiggins, D., \& Netuveli, R. D. (2010). Examining resilience of quality of life in the face of health-related and psychosocial adversity at older ages: What is 'right' about the way we age? The Gerontologist, 50(1), 36-47.

Munro, I., \& Edward K. L., (2008). The lived experience of gay men caring for others with HIV/AIDS: Resilient coping skills. International Journal of Nursing Practice, 14, 122-128.

Newman, R. (2005). APA's resilience initiative. Professional Psychology: Research and Practice, 36(3), 227-229.

Ong, A. D., Zautra, A. J., \& Carrington Reid, M. (2010). Psychological resilience predicts decreases in pain catastrophizing through positive emotions. Psychology and Aging, 25(3), 516-523. 
Patterson, G. T. (2003). Examining the effects of coping and social support on work and life stress among police officers. Journal of Criminal Justice, 31, 215-226.

Pole, N., Kulkarni, N., Bernstein, A., \& Kaufmann, G. (2006). Resilience in retired officers. Traumatology, 12(3), 207-216.

Pooley, J.A. \& Cohen, L. (2010). Resilience: A definition in context. Australian Community Psychologist, 22(1), 30-37.

Pooley, J.A., Cohen, L., O’Connor, M. \& Taylor, M (2012). Posttraumatic Stress and posttraumatic growth and their relationship to coping and self-efficacy in northwest Australian cyclone communities. Psychological Trauma: Theory, Research, Practice and Policy. doi: 10.1037/a0028046

Prati, G., \& Pietrantoni, L. (2010). Risk and resilience factors among Italian municipal police officers exposed to critical incidents. Journal of Police and Criminal Psychology, 25, 27-33.

Richardson, G. E. (2002). The metatheory of resilience and resiliency. Journal of Clinical Psychology, 58(3), 307-321.

Roger, D., Jarvis G., \& Najarian, B. (1993). Detachment and coping: The construction and validation of a new scale for measuring coping strategies. Personality and Individual Differences, 15(6), 619-626. 
Table 1

Frequencies of Demographic Variables

\begin{tabular}{lll}
\hline Variable & Frequency & Percentage \\
\hline Age (years) & & \\
$18-35$ & 75 & 26.3 \\
$36-45$ & 113 & 39.7 \\
$46+$ & 97 & 34.0 \\
Total & 285 & 100 \\
& & \\
Length of Service (years) & & \\
$1-10$ & 101 & 35.4 \\
$11-20$ & 69 & 24.2 \\
$21+$ & 115 & 40.4 \\
Total & 285 & 100 \\
& & \\
Rank & & \\
Constables & 87 & 30.5 \\
Senior Constable & 100 & 35.1 \\
Sergeant & 83 & 29.1 \\
Higher Ranks & 15 & 5.3 \\
Total & 285 & 100 \\
\hline
\end{tabular}

Table 2

Summary of Multiple Regression Analysis for Variables Predicting Resilience

\begin{tabular}{lllll}
\hline Variables & $B$ & $S E B$ & $\beta$ & \\
\hline Rational Coping & .75 & .18 & $.32^{*}$ & \\
Emotional Coping & -1.2 & -.41 & $-.41^{*}$ & \\
Detached Coping & -.29 & -.11 & -.11 & \\
Avoidance Coping & -.40 & -.11 & -.11 & \\
Psych functioning & -.16 & -.05 & -.05 & .44 \\
$R^{2}$ & & & & $44.23^{*}$ \\
$F$ & & & &
\end{tabular}

Table 3

Means and Standard Deviations of Resilience Scores for Demographic Variables

\begin{tabular}{lcc}
\hline & $M$ & $S D$ \\
\hline Length of Service & & \\
1-10 years & $135.83^{*}$ & 13.46 \\
11-20 years & $127.25^{*}$ & 16.79 \\
21 + years & $129.24^{*}$ & 15.24 \\
& & \\
Rank & & \\
Constables & $136.38^{*}$ & 13.24 \\
Snr Constables & $127.30^{*}$ & 16.25 \\
Sergeants & $129.89^{*}$ & 14.91 \\
Higher Ranks & 133.00 & 17.01 \\
& & \\
\hline
\end{tabular}




\begin{tabular}{lcc}
\hline Age & & \\
$18-35$ years & $136.23^{*}$ & 13.68 \\
$36-45$ years & $130.28^{*}$ & 14.89 \\
$46+$ years & $128.07^{*}$ & 16.40 \\
\hline Note: ${ }^{*}$ mean difference is significant at the 0.05 level
\end{tabular}

Table 4

Means, Standard Deviations and Significance of Coping Style Scores for Gender

\begin{tabular}{llcccc}
\hline Coping Style & Gender & $M$ & $S D$ & $t$ & $p$ \\
\hline Rational Coping & Male & 45.97 & 6.59 & & \\
& Female & 44.93 & 6.25 & & \\
Detached Coping & Male & $38.83^{*}$ & 5.63 & & \\
& Female & $37.07^{*}$ & 5.69 & 2.07 & .039 \\
& & & & & \\
Emotional Coping & Male & $25.97^{*}$ & 5.39 & & \\
& Female & $27.69^{*}$ & 4.88 & -2.16 & .032 \\
& & & & & \\
Avoidance Coping & Male & 25.09 & 4.18 & & \\
& Female & 26.02 & 3.96 & & \\
\hline
\end{tabular}

${ }^{*} \mathrm{p}<.05$

Table 5

Means, Standard Deviations and Significance of Coping Style Scores for Length of Service

\begin{tabular}{|c|c|c|c|c|c|}
\hline Coping Style & Length of Service & $M$ & $S D$ & $F$ & $p$ \\
\hline \multirow[t]{3}{*}{ Rational Coping } & $1-10$ years & $46.74 *$ & 5.80 & & \\
\hline & $11-20$ years & $43.42 *$ & 6.37 & & \\
\hline & $21+$ years & $46.31 *$ & 6.92 & 6.21 & .002 \\
\hline \multirow[t]{3}{*}{ Detached Coping } & $1-10$ years & $39.32 *$ & 5.42 & & \\
\hline & $11-20$ years & $36.49 *$ & 5.16 & & \\
\hline & $21+$ years & $38.96 *$ & 5.95 & 5.93 & .003 \\
\hline \multirow[t]{3}{*}{ Emotional Coping } & $1-10$ years & $24.67 *$ & 4.43 & & \\
\hline & $11-20$ years & $27.25 *$ & 5.68 & & \\
\hline & $21+$ years & $27.17 *$ & 5.54 & 7.69 & .001 \\
\hline \multirow[t]{3}{*}{ Avoidance Coping } & $1-10$ years & $23.97 *$ & 3.49 & & \\
\hline & $11-20$ years & $25.88 *$ & 4.31 & & \\
\hline & $21+$ years & $26.04 *$ & 4.33 & 8.11 & .000 \\
\hline
\end{tabular}

Note: $*$ mean difference is significant at the 0.05 level 
Table 6

Mean, Standard Deviations and Significance of Coping Style Scores for Rank

\begin{tabular}{llllll}
\hline Coping Style & Rank & \multicolumn{1}{c}{$M$} & $S D$ & $F$ & $p$ \\
\hline Rational Coping & Constables & $46.71^{*}$ & 5.91 & & \\
& Senior Constables & $43.99^{*}$ & 6.72 & & \\
& Sergeants & 46.35 & 6.78 & & .004 \\
& Higher Ranks & $48.87^{*}$ & 4.50 & 4.60 & \\
& & & & & \\
Detached Coping & Constables & $39.36^{*}$ & 5.46 & & \\
& Senior Constables & $37.04^{*}$ & 5.29 & & .015 \\
& Sergeants & 39.05 & 6.26 & & \\
& Higher Ranks & 40.00 & 4.29 & 3.57 & \\
& & & & & \\
Emotional Coping & Constables & $24.69^{*}$ & 4.48 & & \\
& Senior Constables & $27.19^{*}$ & 5.45 & & \\
& Sergeants & $26.96^{*}$ & 5.61 & & \\
& Higher Ranks & 26.13 & 5.73 & 4.15 & \\
& & & & & \\
Avoidance Coping & Constables & $23.78^{*}$ & 3.47 & & \\
& Senior Constables & $26.45^{*}$ & 4.20 & & \\
& Sergeants & $25.48^{*}$ & 4.23 & & \\
& Higher Ranks & 24.87 & 4.52 & 6.98 & \\
\hline
\end{tabular}

Note: * mean difference is significant at the 0.05 level

Table 7

Mean, Standard Deviations and Significance of Coping Style Scores for Age

\begin{tabular}{llllll}
\hline Coping Style & Age (years) & $M$ & $S D$ & $F$ & $p$ \\
\hline Rational Coping & $18-35$ & $47.16^{*}$ & 6.00 & & \\
& $36-45$ & $44.54^{*}$ & 6.13 & & \\
& $46+$ & 46.11 & 7.14 & 3.93 & .021 \\
Detached Coping & $18-35$ & 39.51 & 5.40 & & \\
& $36-45$ & 37.85 & 5.29 & & \\
& $46+$ & 38.45 & 6.23 & & \\
Emotional Coping & $18-35$ & $25.19^{*}$ & 4.53 & & \\
& $36-45$ & 26.36 & 5.25 & & \\
& $46+$ & $27.26^{*}$ & 5.83 & 3.27 & \\
& & & & & \\
Avoidance Coping & $18-35$ & $24.21^{*}$ & 3.52 & & \\
& $36-45$ & 25.38 & 4.05 & & \\
& $46+$ & $25.96^{*}$ & 4.56 & 3.90 & \\
\hline
\end{tabular}

Note: * mean difference is significant at the 0.05 level 